\title{
Mechanical properties and formation mechanisms of a wire of single gold atoms
}

\author{
Rubio-Bollinger, G.; Bahn, Sune Rastad; Agrait, N.; Jacobsen, Karsten Wedel; Vieira, S.
}

Published in:

Physical Review Letters

Link to article, DOI:

10.1103/PhysRevLett.87.026101

Publication date:

2001

Document Version

Publisher's PDF, also known as Version of record

Link back to DTU Orbit

Citation $(A P A)$ :

Rubio-Bollinger, G., Bahn, S. R., Agrait, N., Jacobsen, K. W., \& Vieira, S. (2001). Mechanical properties and formation mechanisms of a wire of single gold atoms. Physical Review Letters, 87(2), 026101.

https://doi.org/10.1103/PhysRevLett.87.026101

\section{General rights}

Copyright and moral rights for the publications made accessible in the public portal are retained by the authors and/or other copyright owners and it is a condition of accessing publications that users recognise and abide by the legal requirements associated with these rights.

- Users may download and print one copy of any publication from the public portal for the purpose of private study or research.

- You may not further distribute the material or use it for any profit-making activity or commercial gain

- You may freely distribute the URL identifying the publication in the public portal 


\title{
Mechanical Properties and Formation Mechanisms of a Wire of Single Gold Atoms
}

\author{
G. Rubio-Bollinger, ${ }^{1}$ S. R. Bahn, ${ }^{2}$ N. Agraï, ${ }^{1}$ K. W. Jacobsen, ${ }^{2}$ and S. Vieira ${ }^{1}$ \\ ${ }^{1}$ Laboratorio de Bajas Temperaturas, Departamento Física de la Materia Condensada C-III, \\ Instituto Universitario de Ciencia de Materiales "Nicolás Cabrera," \\ Universidad Autónoma de Madrid, E-28049 Madrid, Spain \\ ${ }^{2}$ Center for Atomic-scale Materials Physics, Department of Physics, Technical University of Denmark, \\ DK-2800 Lyngby, Denmark
}

(Received 8 December 2000; published 20 June 2001)

\begin{abstract}
A scanning tunneling microscope supplemented with a force sensor is used to study the mechanical properties of a novel metallic nanostructure: a freely suspended chain of single gold atoms. We find that the bond strength of the nanowire is about twice that of a bulk metallic bond. We perform ab initio calculations of the force at chain fracture and compare quantitatively with experimental measurements. The observed mechanical failure and nanoelastic processes involved during atomic wire fabrication are investigated using molecular dynamics simulations, and we find that the total effective stiffness of the nanostructure is strongly affected by the detailed local atomic arrangement at the chain bases.
\end{abstract}

DOI: 10.1103/PhysRevLett.87.026101

Understanding the mechanical properties of nanostructures is essential for the atomic-scale manipulation and modification of materials, which behave qualitatively different at the nanoscale than at larger dimensions. Mechanical properties of atomic-sized metallic contacts between surface asperities have been studied experimentally using scanning tunneling microscopy (STM) and related techniques [1,2] and theoretically in molecular dynamics (MD) simulations [3-6]. It has been recently shown that it is possible to extract from a previously fabricated nanocontact stable wires of single gold atoms [7,8], in some cases up to seven atoms in length, which are freely suspended between two gold electrodes. The conductance of these onedimensional conductors is close to one quantum unit of conductance $G_{0}=2 e^{2} / h$ (where $e$ is the charge on an electron and $h$ is Planck's constant), because electron transport proceeds through one single quantum conductance channel which is almost completely open [9]. A very recent experimental study of gold nanocontacts at room temperature using transmission electron microscopy shows that nanowires with a last conductance plateau close to $G_{0}$ are one atom thick [10].

We have developed a specific STM supplemented with a force sensor in order to measure the mechanical properties of these nanostructures. The inset in Fig. 1a gives a schematic idea of the experimental setup. The suspended gold nanowire is fabricated between an STM gold tip and a cantilever (cylindrical gold wire, $0.125 \mathrm{~mm}$ diameter, $2 \mathrm{~mm}$ length, $>99.99 \%$ purity) and its conductance measured applying a fixed bias voltage of $10 \mathrm{mV}$. The experiment is performed at $4.2 \mathrm{~K}$. The force during fabrication and breaking of the wire is obtained measuring the cantilever displacement using an auxiliary STM, working in constant tunneling current mode, which follows the force induced displacement of the cantilever free end with picometer resolution. In order to avoid mechanical instabilities related to the softness of the force sensor [5] and to have
PACS numbers: 68.65.La, 62.25.+g, 68.37.Ef, 73.40.Jn

a rigid setup we have used a cantilever whose spring constant is very large $(380 \mathrm{~N} / \mathrm{m})$ compared to the expected nanostructure effective elastic constant which is 2 orders of magnitude smaller.
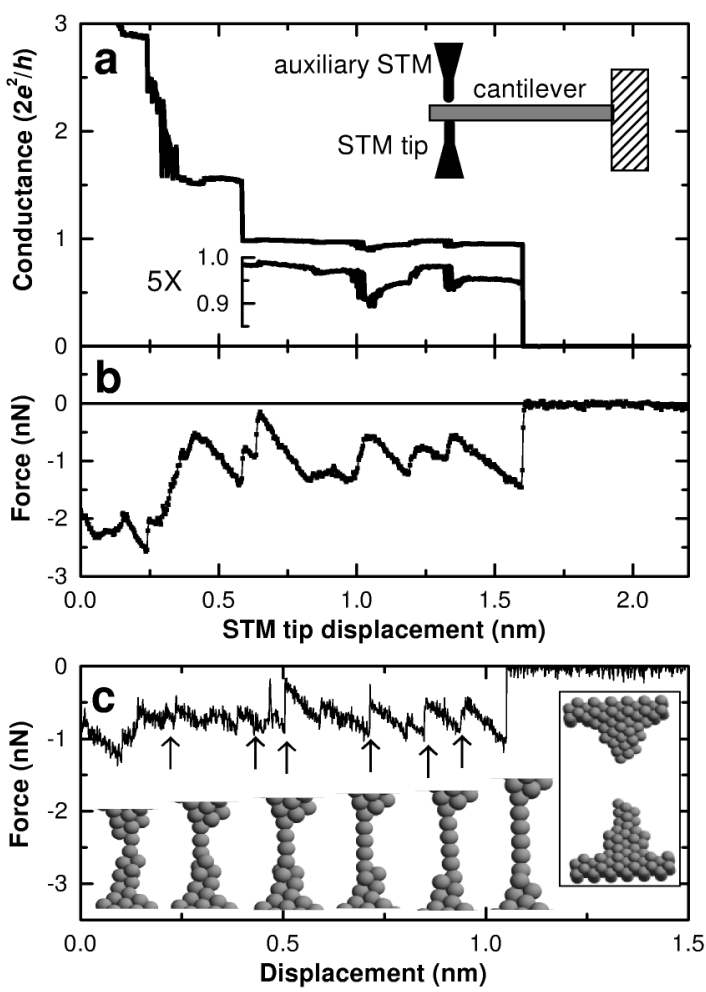

FIG. 1. Simultaneous conductance (a) and force (b) measurements during chain fabrication and breaking. The conductance in the last plateau has been zoomed to show detailed variations. Inset: schematic drawing of the experimental setup. (c) Calculated force during the MD simulation. Arrows indicate where a new atom pops into the chain and snapshots of the structure at these positions are shown. 
An example of simultaneous measurement of conductance and force during fabrication of a gold nanowire is shown in Fig. 1. The experiment starts from a previously formed nanoconstriction which is then stretched at a constant speed $(0.5 \mathrm{~nm} / \mathrm{s})$. The conductance curve displays a steplike behavior down to a value close to one conductance quantum $G_{0}$, which corresponds to a one-atom contact of gold [11], and the simultaneously recorded force curve shows a sawtoothlike signal decreasing in amplitude in a sequence of elastic stages separated by sudden force relaxations related to atomic rearrangements in the nanocontact. This mechanical behavior was predicted in early molecular dynamics simulations [3], and the correspondence between sharp conductance jumps and sudden force relaxations was observed experimentally at room temperature down to contacts consisting of one single atom [1]. The connection between conductance and force has also been addressed in several theoretical studies $[4-6,12]$. In the experiment shown in Fig. 1, the one-atom contact of gold is further stretched a distance of about $1 \mathrm{~nm}$ while the conductance remains close to $2 e^{2} / h$, which signals the formation of a chain of about four atoms that finally breaks [7]. However, in contrast to the behavior observed in metallic nanoconstrictions, the conductance remains almost constant while the force shows large irreversible relaxations between linear stages. Note that there are small conductance jumps related to force relaxations, but their magnitude is much smaller than $2 e^{2} / h$.

In order to gain some insight into the observed mechanical behavior during the formation of an atomic chain we have performed molecular dynamics simulations using effective medium theory (EMT) $[13,14]$ to describe the interatomic interactions. The simulation starts from a nanocontact cut out of an fcc crystal with a cross section at the narrowest region corresponding to 5-10 atomic cross sections. Keeping the temperature at $4 \mathrm{~K}$ the nanocontact is stretched at a rate of $2 \mathrm{~m} / \mathrm{s}$. The simulated strain rate is thus much higher than the experimental one leaving less time for thermally activated processes to occur. Upon stretching, a chain of single gold atoms is in some cases formed by extraction of atoms from the neighboring electrodes into the chain. Snapshots from one such example are shown in Fig. 1c together with the force curve obtained from the simulation. The force curve exhibits a sawtooth shape during the chain formation as seen in the experiments. In the stages with a linearly growing tensile force the nanocontact is elastically stretched, while at the force jumps, abrupt atomic rearrangements occur. Some of the force jumps (generally speaking, the larger ones) correspond to the incorporation of an extra atom into the bridging atomic chain while other relaxations come from atomic rearrangements occurring in the electrode region close to the chain. As long as the force required to rearrange the atoms in the electrodes is smaller than the breaking force, the chain can grow in length. Note that in both the experimental and theoretical curves mechanical relaxations take place at force values smaller than the final breaking force.

We have experimentally observed that the force at which the atomic chain breaks exhibits a very stable value of over 200 nanowire breaking experiments similar to the one shown in Fig. 1, and is independent of chain length. All these nanowires have a conductance close to $G_{0}$, and therefore correspond to one-atom thick wires. We obtain a narrow distribution for the breaking force value centered at $1.5 \mathrm{nN}$. We estimate an accuracy in the force measurement of about $20 \%$ so this leads to a value of $1.5 \pm 0.3 \mathrm{nN}$ for the force needed to break one single bond in the chain. This allows for a stringent test of our knowledge of the metallic bond strength in such low dimensional systems.

In order to carry out a quantitative comparison between theory and experiment we have performed density functional theory (DFT) [15] calculations on small idealized contacts. The DFT calculations have been performed on periodically repeated supercells of different gold chain structures as depicted in Fig. 2b. Electronic exchange and correlation effects are described within the generalized gradient approximation [16], and complete structural relaxation is performed for each configuration studied $[17,18]$.

The results are shown in Fig. $2 b$ where the energy as a function of length of the wire is plotted. We find the breaking force as the slope of the last segment before breaking. The values found are (in $\mathrm{nN})(A)$ 1.62, $(B)$ 1.65, $(C)$ 1.65,
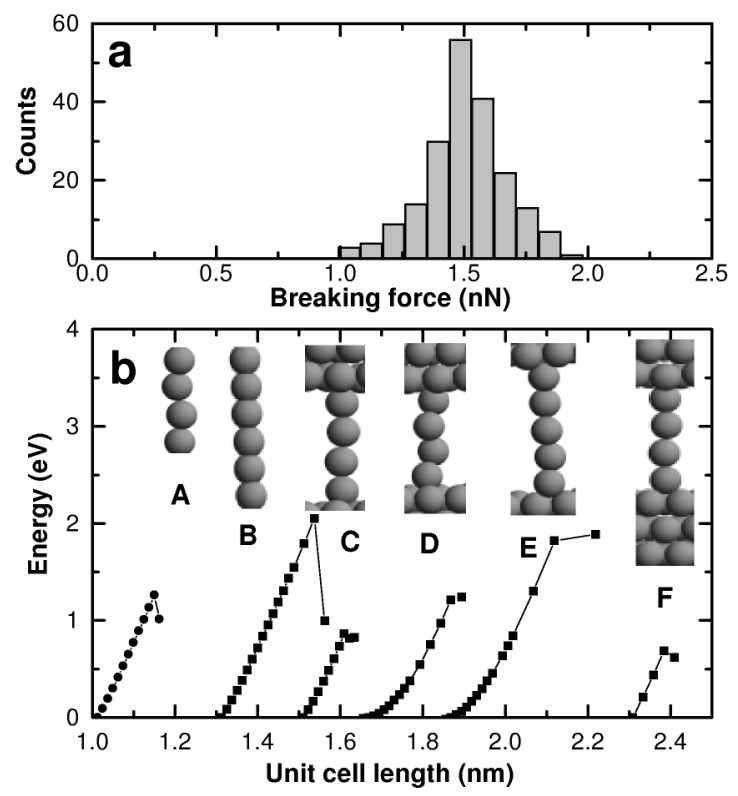

FIG. 2. (a) Histogram of force needed to break the atomic chain, from a set of 200 chain preparation experiments. Note also that only atomic chains whose conductance is close to $G_{0}$ are considered in the histogram. (b) The energy close to breaking for various structures as calculated within density functional theory. The resulting force at the break point is between 1.55 and $1.68 \mathrm{nN}$. The various supercells used in the calculation are shown above each curve. The curves $(B)$ and $(E)$ have been shifted 0.2 units to the left and right, respectively, for clarity. 
(D) $1.55,(E) 1.68$, and $(F)$ 1.58. We thus find a value close to $1.6 \mathrm{nN}$ regardless of the connection of chain to a substrate slab. The lowest value $(1.55 \mathrm{nN})$ is obtained for $(D)$ while the highest $(1.68 \mathrm{nN})$ is seen in the very similar structure of $(E)$. From this we conclude that the influence from the slabs is marginal, in good agreement with the narrow distribution found in the experiment. The value of $1.6 \mathrm{nN}$ is very close to the experimentally observed average value of $1.5 \mathrm{nN}$. However, it should be mentioned that the exact value depends on the approximation for the exchange-correlation energy. Using the RPBE approximation [19] we, for example, estimate a breaking force of $1.4 \mathrm{nN}$. Similar calculations have been used by other authors to investigate the stability and morphology of the chains [20-24]. We note that the observed breaking force $(1.5 \mathrm{nN})$ is considerably higher than what would be expected for individual bonds in bulk gold. An estimate of the maximal force per bond from bulk modulus and cohesive energy considerations results in values of only $0.8-0.9 \mathrm{nN}$ [25]. This is confirmed by direct density functional equation-of-state calculations where we find a maximal force per bulk bond of $0.71 \mathrm{nN}$. Thus the experiments give direct evidence that bonds of low coordinated metal atoms are considerably stronger than bonds in the bulk. The EMT simulations give an average value for the force at breaking somewhat smaller (around $1.0 \mathrm{nN}$ ) than what is seen experimentally and in the DFT calculations. This quantitative discrepancy is not surprising since the EMT potential does not include shell effects [13,14].

In order to understand the nanoelastic properties of the chain/electrode system we have analyzed the elastic stages in the molecular dynamics simulations in more detail. In Fig. 3a we show the relative displacement of the atoms both in the chain and the nearby electrodes during one of the elastic stages. As can be seen from the figure the largest atomic displacements take place in the electrodes, not in the chain itself. This is due to a combination of two effects. One is the electronic effect described above, which makes the bonds in the chain stronger than the bonds in the more bulklike electrodes. The other effect is geometrical. The atoms in the electrodes are sitting in arrangements where the breaking of the bonds can proceed not through direct radial stretching but rather through a more concerted motion of the atoms, giving longer paths and hence smaller forces. This also plays a role for the fact that the electrode is much softer than the bulk. The consequence is the peculiar feature of the microscopic system that thinner is actually stronger in contrast to what is observed in macroscopic systems. For the particular structure shown the effective elastic stiffness of the chain is in fact 5 times larger than that of the electrodes.

The experimental indication of this point comes from studying the slope of the force curves at the elastic stages. Figure $3 b$ shows a plot of the slope of the force curve in the last elastic deformation stage, just before chain rupture, as a function of the length of the chain for a set
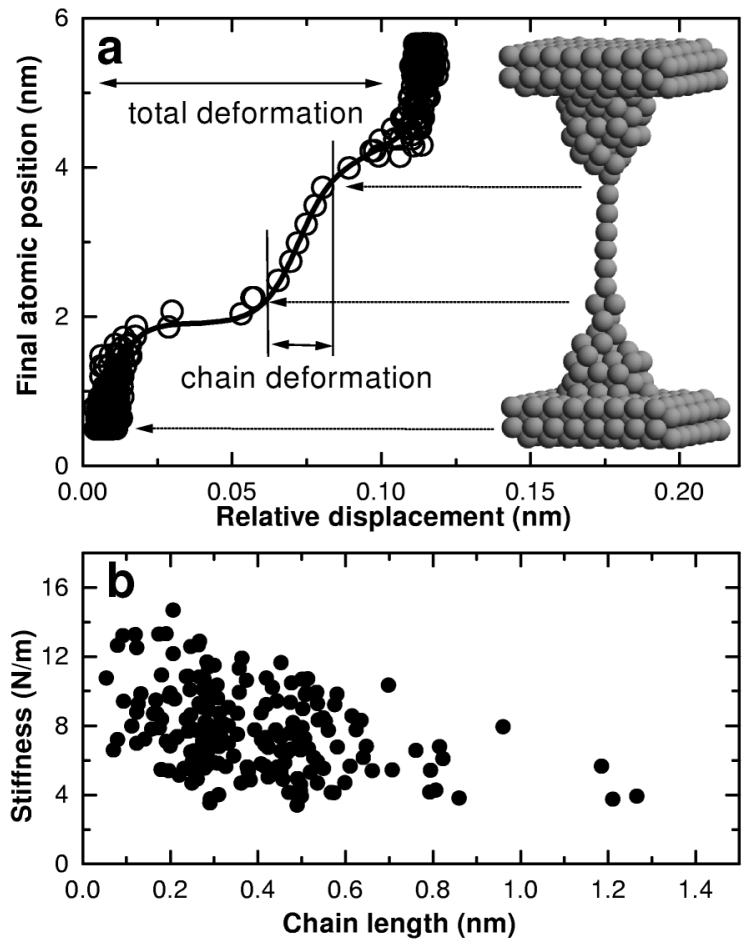

FIG. 3. (a) The relative displacement of each atom in the structure (abscissa) during an elastic deformation stage in the MD simulation. The ordinate gives the position of each atom along the chain axis. To the right is shown the configuration halfway through the stage. (b) Experimental values of the stiffness of the nanostructure just before rupture as a function of chain length.

of 200 nanocontact stretching experiments similar to that shown in Fig. 1. The data for short chains are scattered because the elastic stage slope in the force curve is mainly determined by the compliance of the neighboring electrodes, and this is quite variable depending on the exact atomic configuration of the atoms in the electrodes near the chain ends. However, for longer chains there is less scattering because the elastic constant of the chain becomes comparable to that of the electrodes. Consequently the strain in the nanowire itself cannot be directly related to relative STM tip displacement. In particular, for short chains tip displacement results mostly in force biasing.

Atomic chains of gold are exceptionally simple because they have a single conductance channel which is almost completely open irrespective of their length. Atomic wires of other metals could have different electrical and mechanical properties due to their more complex electronic structure, and detection of chain formation could be more difficult because their conductance might not be necessarily close to $1 G_{0}$. The observation that low coordinated atoms have stronger bonds than atoms in the bulk is quite general, but the exact magnitude of this effect could have a dramatic impact on the possibility of formation of chains in other materials. Further experiments and calculations will be necessary to elucidate these points. 
G.R.-B., N.A., and S.V. were supported by CICyT. CAMP is sponsored by the Danish National Research Foundation.

[1] G. Rubio, N. Agraït, and S. Vieira, Phys. Rev. Lett. 76, 2302 (1996).

[2] A. Stalder and U. Dürig, Appl. Phys. Lett. 68, 637 (1996).

[3] U. Landman, W. D. Luedke, N. A. Burnham, and R. J. Colton, Science 263, 454 (1990).

[4] T. N. Todorov and A. P. Sutton, Phys. Rev. Lett. 70, 2138 (1993).

[5] M. R. Sørensen, M. Brandbyge, and K. W. Jacobsen, Phys. Rev. B 57, 3283 (1998).

[6] C. A. Stafford, D. Baeriswyl, and J. Burki, Phys. Rev. Lett. 79, 2863 (1997).

[7] A. I. Yanson, G. Rubio-Bollinger, and H. E. van den Brom, N. Agraï, and J. M. van Ruitenbeek, Nature (London) 395, 783 (1998).

[8] H. Ohnishi, Y. Kondo, and K. Takayanagi, Nature (London) 395, 780 (1998).

[9] E. Scheer et al., Nature (London) 394, 154 (1998).

[10] V. Rodrigues, T. Fuhrer, and D. Ugarte, Phys. Rev. Lett. 85, 4124 (2000).

[11] N. Agraït, J. G. Rodrigo, and S. Vieira, Phys. Rev. B 47, 12345 (1993).

[12] A. Nakamura, M. Brandbyge, L. B. Hansen, and K. W. Jacobsen, Phys. Rev. Lett. 82, 1538 (1999).

[13] K. W. Jacobsen, J. K. Nørskov, and M. J. Puska, Phys. Rev. B 35, 7423 (1987).
[14] K. W. Jacobsen, P. Stoltze, and J. K. Nørskov, Surf. Sci. 366, 394 (1996), and references therein.

[15] P. Hohenberg and W. Kohn, Phys. Rev. 136, B864 (1964).

[16] J. P. Perdew, J. A. Chevary, S. H. Vosko, K. A. Jackson, M. R. Pederson, D. J. Singh, and C. Fiolhais, Phys. Rev. B 46, 6671 (1992).

[17] We use nonlocal ultrasoft pseudopotentials [18] to describe the ion cores, and the wave functions are expanded in plane waves with kinetic energies up to $25 \mathrm{Ry}$. The first Brillouin zone is sampled with nine special $k$ points. The programs used for the calculations are publicly available at http://www.fysik.dtu.dk/CAMPOS.

[18] D. Vanderbilt, Phys. Rev. B 41, 7892 (1990).

[19] B. Hammer, L. B. Hansen, and J. K. Nørskov, Phys. Rev. B 59, 7413 (1999).

[20] J. A. Torres et al., Surf. Sci. 426, 441 (1999).

[21] D. Sánchez-Portal et al., Phys. Rev. Lett. 83, 3884 (1999).

[22] M. Okamoto and K. Takayanagi, Phys. Rev. B 60, 7808 (1999).

[23] H. Hakkinen, R. N. Barnett, and U. Landman, J. Phys. Chem. B 103, 8814 (1999).

[24] L. De Maria and M. Springborg, Chem. Phys. Lett. 323, 293 (2000).

[25] J. H. Rose et al., Phys. Rev. B 28, 1835 (1983). We use the "universal" suggested binding energy function. The cohesive energy, $E$, as a function of lattice parameter, $a$, is then given by $E(a)=E_{\text {coh }} f\left(\left(a-a_{0}\right) / \ell\right)$, with $f(x)=$ $(1+x) \exp (-x)$, where $\ell$ is a characteristic length. The resulting maximal force per bond is $\left(E_{\mathrm{coh}} B a_{0} / 8 e^{2}\right)^{(1 / 2)}$, where $E_{\text {coh }}$ is the equilibrium cohesive energy $B$ is the bulk modulus, and $a_{0}$ is the equilibrium lattice constant. 